\title{
Projective Display of Document Information by Parametric Sound Beam
}

\author{
Yoshimichi Yonezawa, Hideki Nonaka, \\ Kazunori Itoh and Masami Hashimoto \\ Department of Information Engineering \\ Faculty of Engineering, Shinshu University \\ 500 Wakasato Nagano-shi 380 JAPAN
}

\begin{abstract}
For the input work of sentences by the blind, the location information of cursor is necessary. We have tried to display document information by the sound(voice). The sound we used is secondary one derived from parametric array driven by an ultrasonic range signal. We got the movable sound source using the sound and projecting it on the wall just like torch light. The basic characteristics show the possibility of the document information display by this method.
\end{abstract}

\section{Introduction}

The key-in work is thought to be hopeful work for the blind because they can work as fast as the non-visually handicapped people when they've got used to the work(by training). The system they use is usually make to support the work by the function of tactile one-line braille display or voice confirming system. But, there is no system which transfers the information of two dimensional layout of key-inned document and position of cursor realtimely to an operator, though it is an ordinary function of CRT.

The new system we tried can cover the lack of functions above mentioned by the sound with positional or vocal information. Because the sound source position in free space can be recognized (localized) easily by hearing the sound, there is a possibility to display images by the sound if we make sounds or voices correspond to colors or characters respectively and move the sound source in space. We tried to make sound beam, to project it on the wall and to deflect the beam and got a reflected movable sound source. The sound beam was made by using parametric array effect[1,2].

\section{Principle of Parametric Array}

The parametric array effect we adopted is caused by a nonlinear characteristic of air for acoustic pressure. And for example, an ultrasonic wave (primary) of $40 \mathrm{KHz}$ modulated by $1 \mathrm{KHz}$ sine wave in amplitude makes the secondary one corresponding to $1 \mathrm{KHz}$ wave along the way of propagation of primary wave in air(Fig. 1). 


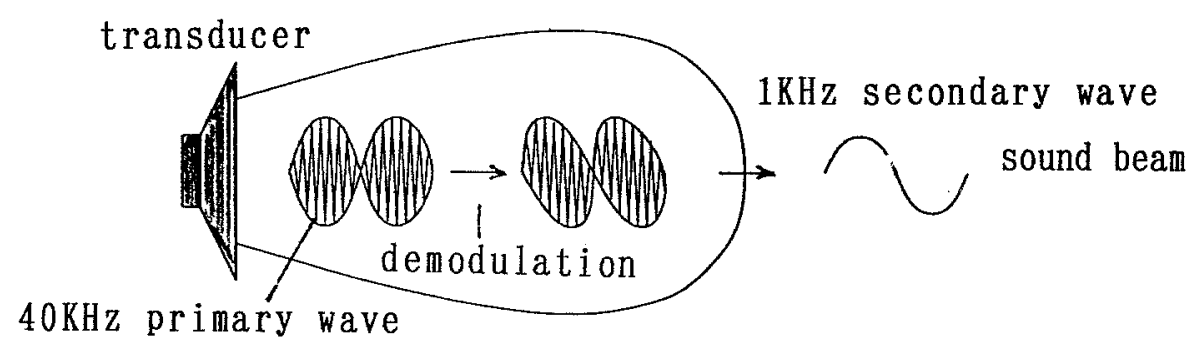

Fig. 1 Principle of parametric array

In this case, the generation points of the secondary wave are localized in the pattern of phased array and then beam-like sound pressure distribution of secondary wave is realized. If we use a voice signal as modulator one, we can get a voice as a secondary wave beam.

We tried to make movable sound source by projecting the beam on the wall (screen) and deflect it two-dimensionally, because the projected and reflected sound is heard as if there is a sound source on the wall. The beam is deflected by two axial servo mechanism controlled by computer(Fig. 2).

\section{Localization Characteristics I (1 Dimensional)}

The transducer used for this system is piezoelectric type and a matrix array of transducers $(4 \times 4)$ is used for primary sound source. The secondary wave derived from parametric array effect has characteristics in frequency as shown in Fig. 3 . The frequency range shows that display sound can be voice or any audible sound. Really, we can hear and message and music from reflective sound source on the screen.

To evaluate the display method, two kinds of screens were made and one dimensional accuracy of localization of the reflective sound (white noise) was measured. Fig. 4 shows the two kinds of screen, one is plane and another is a part of ellipse with two focuses, - one at listener and another at primary sound source (transducer). Fig. 5 is the lattice pattern on the screen used for evaluation of the system, and the listener was asked to answer the recognized position by the $\mathrm{x}, \mathrm{y}$ number of lattice on the screen when the direction of the sound beam is changed on-dimensionally one after another randomly.

Fig. 6 shows the result of the experiment for two kinds of screen. The size of the plotting mark shows the rate of the correct answer for every position as shown under the figure. If the display point is recognized perfectly, the largest marks $(10 / 10)$ are arrayed on the inclined straight line. These results show that higher answer rate is shown on the elliptical screen. 


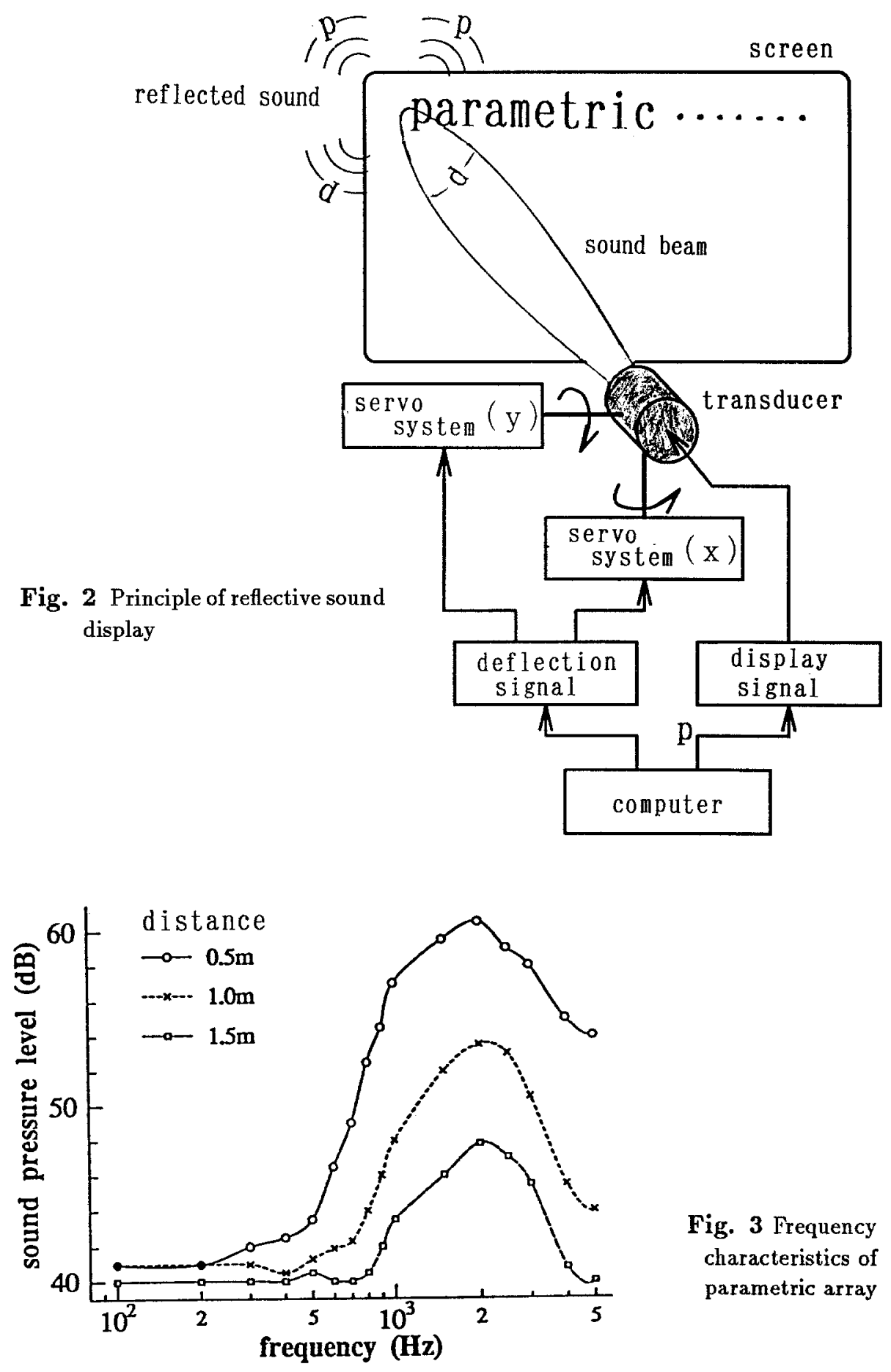




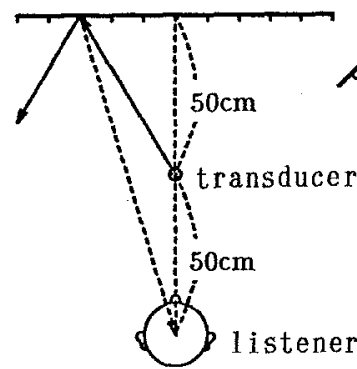

(a) Plane

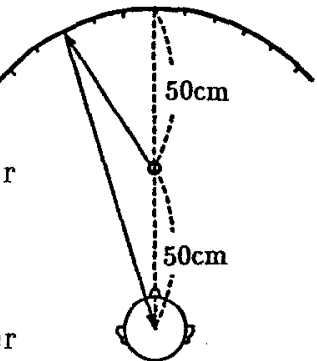

(b) Elliptical

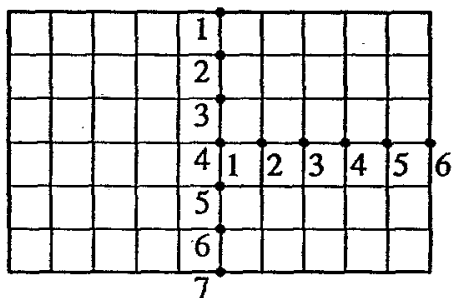

Fig. 4 Two kinds of screen

Fig. 5 Lattice pattern on the screen
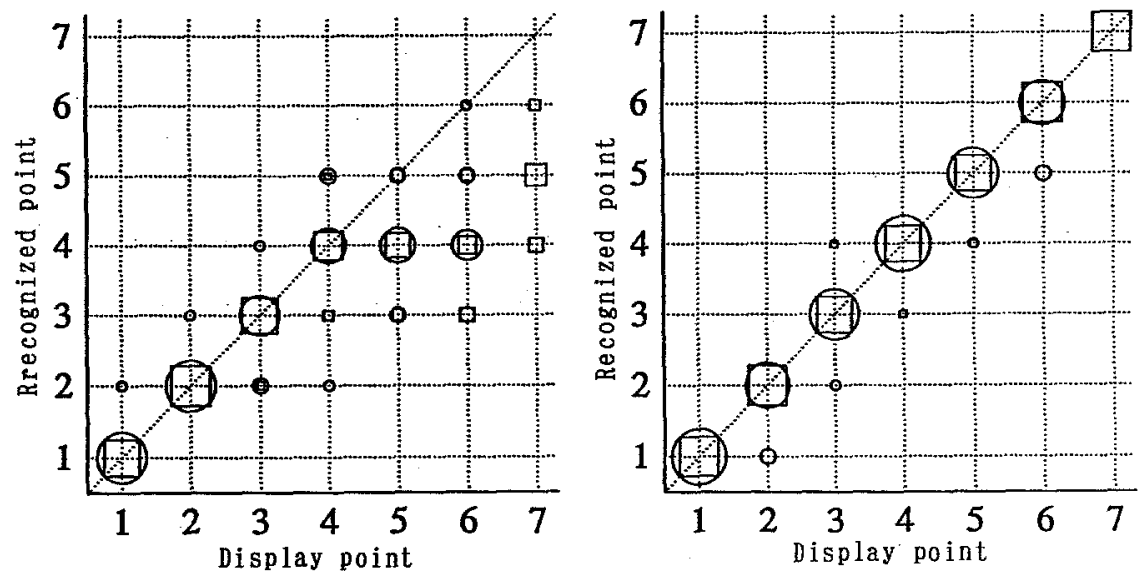

(a) Plane screen

(b) Elliptical screen

Fig. 6 1-dimensional recognition characteristics
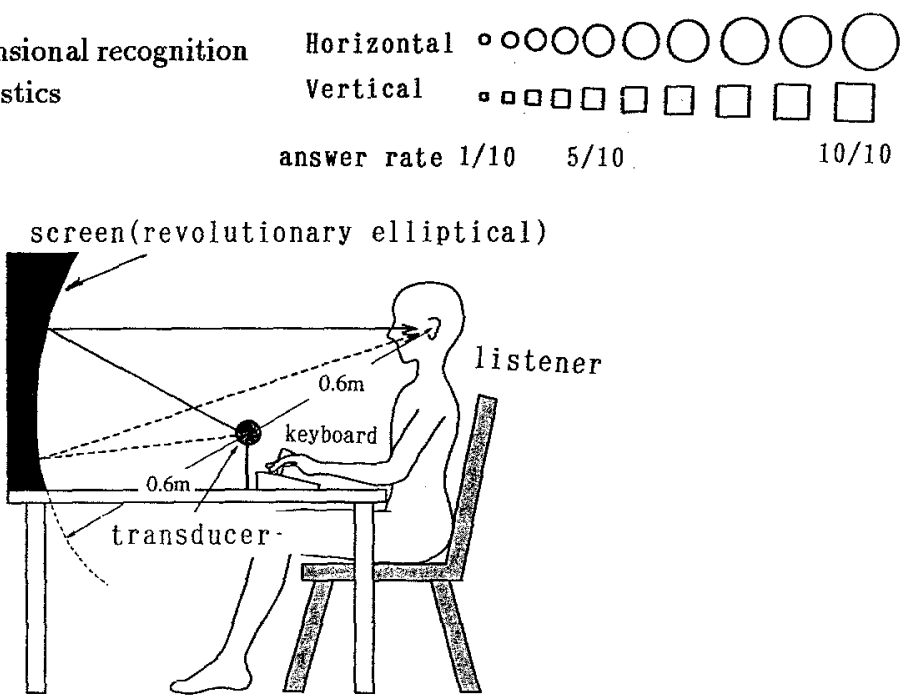

Fig. 7 2-dimensional screen and listener 


\section{Localization Characteristics II (2 Dimensional)}

A two-dimensionally curved screen was made to evaluate the two-dimensional location accuracy. The curved surface of revolutionary ellipse was made based on the result of one dimensional localization accuracy measurement. By this screen any sound beam projected on the screen comes back to listener as shown in Fig. 7.

The localization accuracy is also measured and shown in Fig. 8. The size of round mark shows the rate of correct answers obtained by $1 / 4$ screen (right under, $5 \times 5$ points) as shown under the figure as example. And the size of an inner circle shows pure correct answer rate respectively and that of the outer one corresponds to the answer rate including the case when the point designated by the listener is the neighboring point. The dark circle shows the case of correct answer rate under 40To improve the recognition rate, diplejs $(30 \mathrm{~mm}$ in diameter) are carved on the surface of screen to disperse the reflective beam in 50 degrees (Fig. 9). This makes the wave front of reflective sound more natural and easier to listen to. The result in Fig. 9 shows the improvement in localization by this method. lattice number(horizontal)

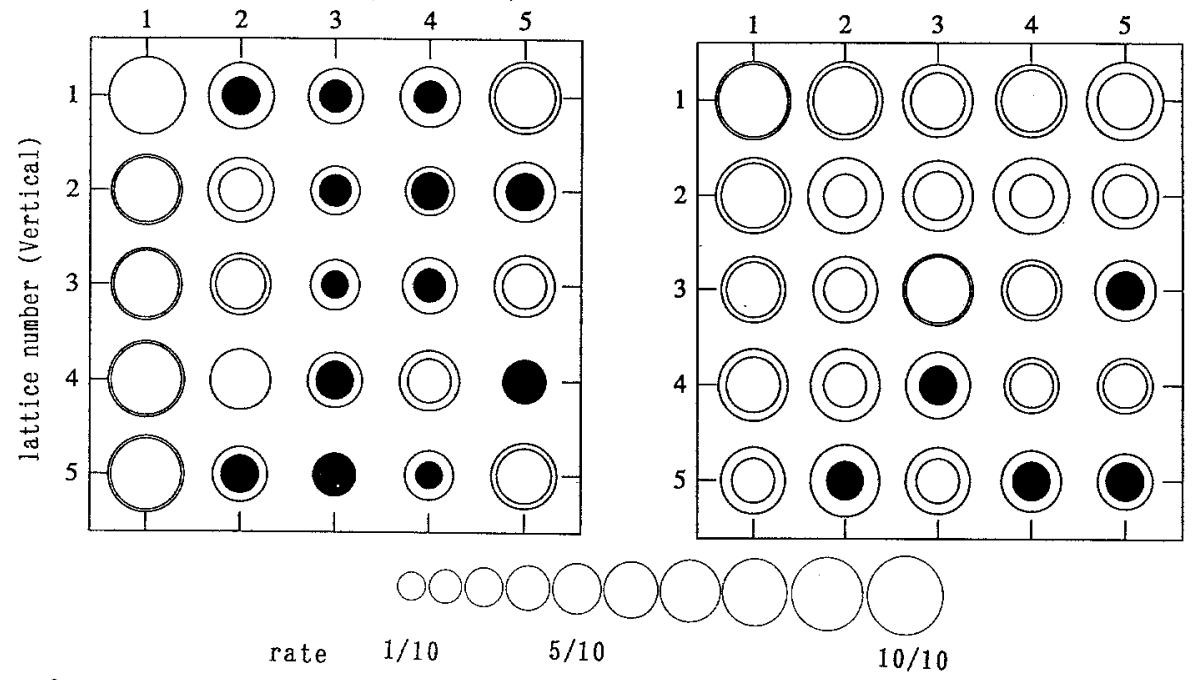

Fig. 8 Localization rate of the flat screen

\section{Conclusion}

Fig. 9 Localization rate of the screen with dimples

A movable sound source was made by formation of audible sound beam by parametric array, by projection on a wall and by deflecting the beam twodimensionally. The screen was formed by the elliptical curve and dimples are added on the screen for better localization.

The movable sound source on the screen can emit voice and any defined special sound (cursor, comma etc.) with locative information. This function is just like CRT and can be a great support for the key-in work of visually handicapped people. 


\section{References}

1. P. J. Westervelt, "Parametric acoustic array", J. Acoust. Soc. Am. 35, 535-537 (1963)

2. M. Yoneyama, J. Fujimoto, Y. Kawamo and Sasabe, "The audio spotlight: An application of nonlinear interaction of sound wave to a new type of loudspeaker design", J. Acoust. Soc. Am. 73, 1522-1536 (1983) 